\title{
Article \\ Activity and Safety of NAB-FOLFIRI and NAB-FOLFOX as First-Line Treatment for metastatic Pancreatic Cancer (NabucCO Study)
}

\author{
Elisa Giommoni ${ }^{1, *}\left(\mathbb{0}\right.$, Evaristo Maiello ${ }^{2}$, Vanja Vaccaro ${ }^{3}$, Ermanno Rondini ${ }^{4}$, Caterina Vivaldi ${ }^{5}$, \\ Giampaolo Tortora ${ }^{6}$, Laura Toppo ${ }^{7}$, Guido Giordano ${ }^{8}$ (1) , Tiziana Pia Latiano ${ }^{2}$, Cinzia Lamperini ${ }^{1}$, \\ Serena Pillozzi ${ }^{1}{ }^{\mathbb{D}}$, Luca Boni ${ }^{9}{ }^{\mathbb{D}}$, Lorenzo Antonuzzo ${ }^{1}$ and Francesco Di Costanzo ${ }^{1, \dagger}$
}

check for

updates

Citation: Giommoni, E.; Maiello, E.; Vaccaro, V.; Rondini, E.; Vivaldi, C.; Tortora, G.; Toppo, L.; Giordano, G.;

Latiano, T.P.; Lamperini, C.; et al Activity and Safety of NAB-FOLFIRI and NAB-FOLFOX as First-Line Treatment for metastatic Pancreatic Cancer (NabucCO Study). Curr. Oncol. 2021, 28, 1761-1772. https:// doi.org/10.3390/curroncol28030164

Received: 8 April 2021

Accepted: 30 April 2021

Published: 8 May 2021

Publisher's Note: MDPI stays neutral with regard to jurisdictional claims in published maps and institutional affiliations.

Copyright: (C) 2021 by the authors Licensee MDPI, Basel, Switzerland. This article is an open access article distributed under the terms and conditions of the Creative Commons Attribution (CC BY) license (https:// creativecommons.org/licenses/by/ $4.0 /)$.
1 Medical Oncology Unit, Careggi University Hospital, 50134 Florence, Italy; cinzia.lamperini@gmail.com (C.L.); serena.pillozzi@unifi.it (S.P.); lorenzo.antonuzzo@unifi.it (L.A.); dicostanzofrancesco@icloud.com (F.D.C.)

2 Medical Oncology Unit, IRCCS Casa Sollievo della Sofferenza, 71013 San Giovanni Rotondo, Italy; e.maiello@libero.it (E.M.); latiano.tiziana@virgilio.it (T.P.L.)

3 Medical Oncology Unit, Istituto Nazionale Tumori Regina Elena, 00144 Roma, Italy; vanja.vaccaro@hotmail.it

4 Oncology Unit, Ospedale Santa Maria Nuova-IRCCS, 42100 Reggio Emilia, Italy; ermanno.rondini@gmail.com

5 Medical Oncology Unit 2, Azienda Ospedaliero Universitaria Pisana, 56126 Pisa, Italy; caterina.vivaldi@gmail.com

6 Medical Oncology Unit, Azienda Ospedaliera Universitaria Integrata, 37134 Verona, Italy; giampaolo.tortora@policlinicogemelli.it

7 Medical Oncology Unit, ASST Cremona, 26100 Cremona, Italy; lauratoppomed@gmail.com

8 Oncology Unit, Ospedale "Sacro Cuore di Gesù" Fatebenefratelli, 82100 Benevento, Italy; giordano.guido81@gmail.com

9 Clinical Trial Coordinating Center, Careggi University Hospital, 50134 Florence, Italy; luca.boni@hsanmartino.it

* Correspondence: giommonie@aou-careggi.toscana.it

+ On behalf of GOIRC (Italian Cooperative Oncology Group for Clinical Research).

Abstract: Background: Relevant improvement in first-line treatment of metastatic pancreatic cancer (mPC) was provided by FOLFIRINOX and by gemcitabine (gem) plus nab-paclitaxel (Nab-p) regimens. Regardless of the first-line treatment survival benefit, most patients survive less than 1 year. Aim: The objectives of this multicenter phase I/II study were to evaluate as first-line chemotherapy (CT) two modified regimens of FOLFIRINOX, replacing either oxaliplatin (Oxa) or irinotecan with Nab-p, in patients with mPC. Methods: The primary objectives of phase 1 were the definition of the dose limit binations, while for phase II they were the characterization of safety and activity of Nab-FOLFIRI and Nab-FOLFOX in mPC. Results: Sixty-three patients received Nab-FOLFIRI or Nab-FOLFOX in phase I. We defined MTD at $120 \mathrm{mg} / \mathrm{m}^{2}$ for Nab-p with FOLFIRI and $160 \mathrm{mg} / \mathrm{m}^{2}$ with FOLFOX. In phase II, we randomized 42 patients for each arm with the following results: (1) overall response rate (ORR) was 31\% for both schedules; (2) a clinical benefit rate (CBR) of $69 \%$ and $71 \%$; (3) 1-year survival was $41 \%$ and 50\%; (4) progression free survival (PFS) was 6 months and 5.6 months; (5) median overall survival (OS) was 10.2 and 10.4 months for Nab-FOLFIRI and Nab-FOLFOX, respectively. (6) Neutropenia was the most common grade $\geq 3$ adverse event in our regimens, significantly lower than that reported for the FOLFIRINOX triplet. Conclusion: Nab-FOLFIRI and Nab-FOLFOX might be hopeful first-line CT options for mPC patients, with promising activity and a good safety profile.

Keywords: metastatic pancreatic cancer; nab-paclitaxel; FOLFIRINOX; dose finding 


\section{Introduction}

PC is the seventh leading cause of cancer death worldwide in both sexes with about 331,000 deaths per year and an estimated 5-year survival rate of about 5\%. Overall, about 53,670 new cases of PC were expected in the USA in 2017, while the annual incidence in the EU is 78,654 where it represents the fourth cause of death by cancer. Fifty percent of new diagnoses are in an advanced or $\mathrm{M}$ stage and more than $70 \%$ of the resected patients will experience a recurrence, with median OS ranging from 4 to 10 months [1-4].

Standard therapy for mPC is $\mathrm{CT}$, which improves survival compared to the best supportive care according to a meta-analysis of seven randomized trials. For more than a decade, gem was the approved single agent treatment, while all other phase III trials failed to improve OS significantly [5-7].

Relevant improvement in the first-line treatment of mPC was provided by FOLFIRINOX (5-fluorouracil (5-FU) (antimetabolite drug), irinotecan (CPT11) (inhibitor of topoisomerase I), and oxaliplatin (Oxa) (exerts its cytotoxic effect through DNA damage)) and by gem (inhibitor of DNA synthesis) plus Nab-p (a microtubule stabilizing drug) regimens [8-12].

In the French trial (PRODIGE 4/ACCORD 11), the FOLFIRINOX combination obtained a better response rate (RR) and OS than gem alone. However, this combination showed significant toxicities with higher rates of grade $\geq 3$ neutropenia with respect to gem alone [8].

Nab-paclitaxel (Nab-p), an albumin-bound formulation of paclitaxel [9], demonstrated a significantly longer OS in association with gem versus gem alone in the MPACT trial [10].

Regardless of the impact of first-line treatment on survival, most patients survive less than 1 year [3]. This poor prognosis highlights the need for new treatment options for $\mathrm{mPC}$.

The Italian Cooperative Oncology Group for Clinical Research (GOIRC) designed a phase I/II trial to evaluate the replacement of either Oxa or CPT11 with Nab-p in the original FOLFIRINOX schedule, obtaining two new regimens: Nab-FOLFIRI (arm A) and Nab-FOLFOX ( $\operatorname{arm} B$ ). We postulated that these regimens would enhance activity with no additional toxicity compared to FOLFIRINOX.

The objectives of the phase $\mathrm{Ib}$ part were to define the dose limiting toxicities (DLTs) and maximum tolerated dose (MTD). In the second part, both schedules were evaluated in a multicenter randomized 1:1 open label phase II trial. The principal end points of phase II were to assess activity and safety of Nab-FOLFIRI and Nab-FOLFOX in patients with mPC as first-line CT.

\section{Materials and Methods}

\subsection{Patients and Study Design}

From February 2014 to October 2015, patients were enrolled from 7 Italian centers for phase I, while phase II patients were enrolled in 8 Italian sites from November 2015 to January 2017.

Eligibility criteria included age $\geq 18$ and $\leq 75$; a histological or cytological diagnosis of $\mathrm{mPC}$ with measurable disease defined according to the Response Evaluation Criteria in Solid Tumor (RECIST version 1.1 guidelines) [13] and no previous treatment for metastatic disease; an Eastern Cooperative Oncology Group (ECOG) PS score of 0 or 1, absence of previous abdominal radiotherapy on target lesions, absence of heart failure or unstable angina, or infarction within 12 months previous to inclusion; adequate organ function, including hematologic (absolute neutrophil count (ANC) $\geq 1.5 \times 10^{9} / \mathrm{L}$, platelets $\geq 100 \times 10^{9} / \mathrm{L}$ ), hepatic (bilirubin $\leq 1.5$ times upper limits of normal (ULN), and renal (creatinine within normal limits $\leq 1.5$ times (ULN)) values. Patients could have endoscopic or radiologic stenting to treat biliary obstructions.

Patients were not eligible if they had endocrine or acinar pancreatic carcinoma, known dihydropyrimidine dehydrogenase (DPD) deficiency, central nervous system metastases, other concomitant cancer, or history of cancer outside of carcinoma in situ of the 
cervix, basal, or squamous cell cancer; current active infections, serious pre-existing medical conditions or serious concomitant systemic disorders, a history of chronic diarrhea or inflammatory disease of the colon or rectum, or sub-occlusion or occlusion not resolved under symptomatic treatment; females who were pregnant or lactating were also excluded; and all subjects deprived of liberty or under guardianship or patients unable to undergo medical tests for geographical, social, or psychological reasons. Prior adjuvant treatment was allowed with tumor recurrence occurred $\geq 6$ months after the last treatment.

Written informed consent was obtained from all patients before every study procedure.

The study was conducted in accordance with Good Clinical Practice (GCP), all regulatory requirements, and the principles of the Declaration of Helsinki. This study was submitted to and approved by the Ethics Committee of the cancer centers involved and also registered in the clinical trial network provided by US NIH (Clinicaltrials.gov NCT02109341).

\subsubsection{Phase I}

Phase I was a non-randomized, open label, two arm dose-finding study for Nab-p in combination with FOLFIRI (l-folinic acid $200 \mathrm{mg} / \mathrm{m}^{2}$ intravenous (iv); 5 -FU bolus $400 \mathrm{mg} / \mathrm{m}^{2}$ iv and $48 \mathrm{~h}$ continuous infusion (ci) $2400 \mathrm{mg} / \mathrm{m}^{2}$ at days 1 and 2; CPT11 $180 \mathrm{mg} / \mathrm{m}^{2}$ iv at day 1) and FOLFOX (1-folinic acid $200 \mathrm{mg} / \mathrm{m}^{2} \mathrm{iv} ; 5-\mathrm{FU}$ bolus $400 \mathrm{mg} / \mathrm{m}^{2}$ iv and $48 \mathrm{~h} \mathrm{ci}$ $2400 \mathrm{mg} / \mathrm{m}^{2}$ at days 1 and 2; oxa $85 \mathrm{mg} / \mathrm{m}^{2}$ iv at day 1) every 2 weeks. The design used was the classical $3+3$ design 13 , with a dose escalation of $10 \mathrm{mg} / \mathrm{m}^{2}$ for each subsequent cohort. DLTs were treatment-related toxicities during the first cycle according to the National Cancer Institute Common Terminology Criteria of Adverse Event (CTCAE) ver 3.0 (https: / / ctep.cancer.gov/protocoldevelopment/electronic_applications/docs/ctcaev3 .pdf, accessed on 4 September 2013). Dose escalation was stopped when one or more patients of a cohort of three patients experienced a DLT, and the dose below was declared MTD by a confirmatory cohort of three more patients.

\subsubsection{Phase II}

In phase II, patients were randomized to receive Nab-p at a dose defined in phase I in association with FOLFIRI (arm A) (l-folinic acid $200 \mathrm{mg} / \mathrm{m}^{2}$ intravenous (iv); 5-FU bolus $400 \mathrm{mg} / \mathrm{m}^{2}$ iv and $48 \mathrm{~h}$ continuous infusion (ci) $2400 \mathrm{mg} / \mathrm{m}^{2}$ at days 1 and 2; CPT11 $180 \mathrm{mg} / \mathrm{m}^{2}$ iv at day 1) and with FOLFOX (arm B) (1-folinic acid $200 \mathrm{mg} / \mathrm{m}^{2} \mathrm{iv} ; 5$-FU bolus $400 \mathrm{mg} / \mathrm{m}^{2}$ iv and $48 \mathrm{~h}$ ci $2400 \mathrm{mg} / \mathrm{m}^{2}$ at days 1 and 2; oxa $85 \mathrm{mg} / \mathrm{m}^{2}$ iv at day 1) every 2 weeks. Patients continued treatment until disease progression or unacceptable toxicity, up to a maximum of 12 cycles.

The modified intention to treat the ITT population consisted of all patients who were randomized and received at least one dose of any component of study treatment. Patients were grouped according to the randomized treatment assignment. Patients treated during phase I were not included in this population. The safety population consisted of all patients who were randomized and received at least one dose of any component of study treatment. The activity was the primary outcome of interest. The response assessment was by computed tomography scan or magnetic resonance imaging (MRI) at baseline and every 8 weeks according to RECIST version 1.0. Clinical benefit rate (CBR) was estimated by dividing the total number of complete responses (CR), partial responses (PR), or stable disease (SD) by the number of patients included into the modified ITT population, and the exact 95\% confidence intervals for each treatment arm were determined. PFS and OS were evaluated using Kaplan-Meier estimates of the median survival times (including 95\% confidence intervals (CI)) and 6 month survival to inform assumptions for use in future trial planning.

\subsubsection{Study Assessment}

Each patient enrolled in the study was evaluated at baseline with a physical exam, determination of ECOG PS, and clinical laboratory tests (blood count, sodium, potassium, alkaline phosphatase, alanine aminotransferase, aspartate aminotransferase, blood urea 
nitrogen, creatinine, glucose, albumin, lactate dehydrogenase, gamma glutamyltransferase, bilirubin). Tumor assessment at baseline was performed using a computed tomography scan or MRI as defined by RECIST.

Response was assessed every 8 weeks during treatment, then every three months during follow-up until progression of disease (PD), consent withdrawal, or death. Assessments included the following parameters: physical examination, clinical symptoms, laboratory or radiological findings, and tumor assessment by CT or MRI. All patients who received at least one dose of a study drug were evaluated for activity and safety. Safety was assessed by the incidence of treatment-related adverse events (AEs) according to CTCAE version 3.0.

Quality of life (QOL) was assessed using the European Organization for Research and Treatment of Cancer Quality of Life Questionnaire C30 (EORTC QLQ-C30, version 3.0). The EORTC QLQ-C30 is a 30 item questionnaire including five functional scales, three symptom scales, one QOL scale, and six single items on common symptoms. The EORTC QLQC30 questionnaire was distributed to the patients at baseline, then every two cycles and at the end of treatment. Questionnaires were scored according to the EORTC instructions.

\section{Results}

NabucCO, a two-part multicenter phase I/II study, assessed as first-line CT two modified regimens of FOLFIRINOX, replacing either oxaliplatin or irinotecan with Nab-p (named Nab-FOLFIRI and Nab-FOLFOX arms), in patients with mPC (Figure 1).

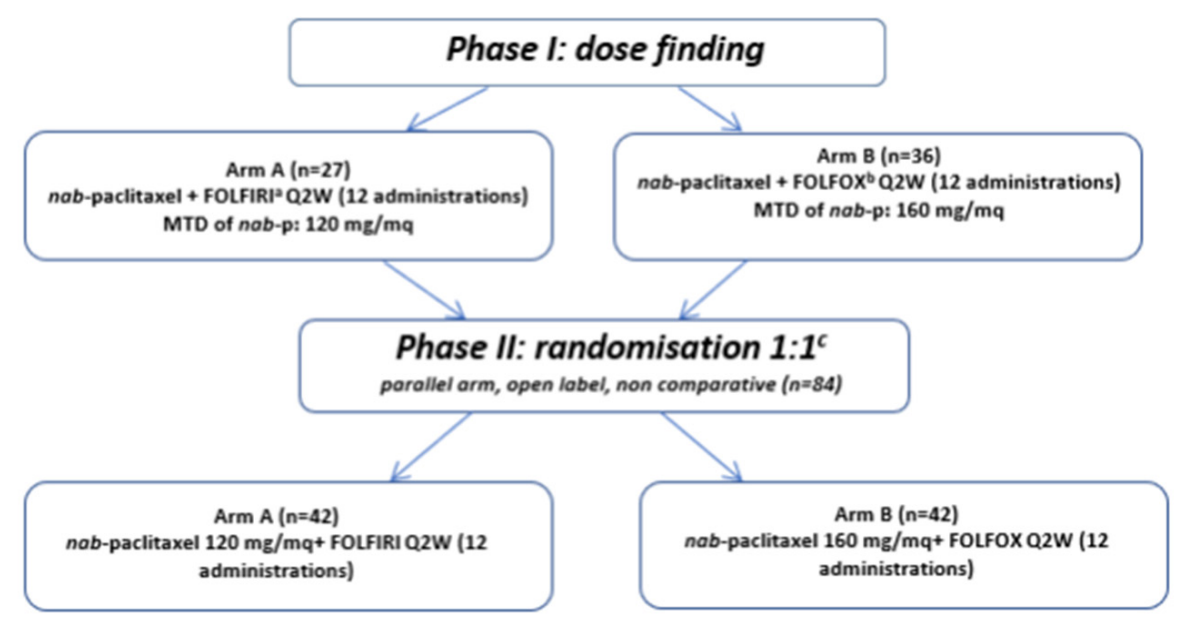

a FOLFIRI: irinotecan $180 \mathrm{mg} / \mathrm{m}^{2}+$ leucovorin $400 \mathrm{mg} / \mathrm{m}^{2}+$ fluorouracil $400 \mathrm{mg} / \mathrm{m}^{2}$ Q2W

bFOLFOX: oxaliplatin $85 \mathrm{mg} / \mathrm{m}^{2}$ + leucovorin $400 \mathrm{mg} / \mathrm{m}^{2}+$ fluorouracil $400 \mathrm{mg} / \mathrm{m}^{2}$ Q2W

' nab-Paclitaxel at recommended dose from Phase I

Figure 1. Flow chart of NabucCO study design.

We sought to develop a regimen that could potentially be better tolerated than FOLFIRINOX in MPC and, hence, could be administered for multiple cycles without the development of severe toxicity. For dose finding (phase I), the primary objectives were the definition of the DLTs and of the MTD of Nab-p in both combinations. For phase II, the primary objectives were further characterization of the safety and, in addition, the evaluation of the activity of Nab-FOLFIRI and Nab-FOLFOX in mPC in terms of ORR.

\subsection{Phase I}

From February 2014 to October 2015, 63 patients were enrolled in 8 Italian centers (on behalf of GOIRC) to receive either Nab-FOLFIRI $(n=27)$ or Nab-FOLFOX $(n=36)$ (Table 1). In the Nab-FOLFIRI arm the median age was 62 years (range 38-75) and 60 years (range 43-74) in the Nab-FOLFOX arm. DLTs during first cycle at the corresponding dose level are listed in Table 2. The MTDs for Nab-p were $120 \mathrm{mg} / \mathrm{m}^{2}$ in association with FOLFIRI and $160 \mathrm{mg} / \mathrm{m}^{2}$ with FOLFOX. The most common adverse events (AEs) of 
any grade, in the Nab-FOLFIRI and Nab-FOLFOX arms, respectively, were the following: neutropenia ( $52 \%$ and $81 \%$ ), anemia $(49 \%$ and $45 \%)$, thrombocytopenia $(11 \%$ and $45 \%)$, nausea (56\% and $47 \%)$, asthenia ( $56 \%$ and $53 \%$ ), diarrhea (70\% and $22 \%)$, liver toxicity $(18 \%$ and $44 \%)$, and peripheral neuropathy $(0 \%$ and $47 \%)$. In particular, the AEs $\geq$ grade 3 were neutropenia ( $45 \%$ and $31 \%$ ), febrile neutropenia ( $4 \%$ and $11 \%)$, anemia $(19 \%$ and $0 \%)$, liver toxicity ( $11 \%$ and $6 \%)$, and asthenia ( $7 \%$ and $8 \%)$. Two patients experienced a peripheral neuropathy of grade 3 (in the Nab-FOLFOX arm), a grade 3 liver function alteration was observed in 3 patients ( 2 with Nab-FOLFIRI treatment and 1 with NabFOLFOX), and a grade 4 toxicity was registered in 2 patients (1 patient for each schedule). The ORR were $18.5 \%(5 / 27)$ and $25 \%(9 / 36)$, respectively, in the two arms.

Table 1. Phase I: patient's characteristics.

\begin{tabular}{|c|c|c|c|c|}
\hline \multirow{2}{*}{ Patient's Characteristics } & \multicolumn{2}{|c|}{ Nab-FOLFIRI (Arm A) } & \multicolumn{2}{|c|}{ Nab-FOLFOX (Arm B } \\
\hline & $N$ & $\%$ & $N$ & $\%$ \\
\hline \multicolumn{5}{|l|}{ Sex } \\
\hline Male & 15 & 56 & 14 & 39 \\
\hline \multicolumn{5}{|l|}{ Female } \\
\hline \multicolumn{5}{|l|}{ Age, years } \\
\hline Median & \multirow{2}{*}{\multicolumn{2}{|c|}{$\begin{array}{c}62 \\
(38-75)\end{array}$}} & \multirow{2}{*}{\multicolumn{2}{|c|}{$\begin{array}{c}60 \\
(42-74)\end{array}$}} \\
\hline Range & & & & \\
\hline \multicolumn{5}{|l|}{ ECOG } \\
\hline 0 & 19 & 70 & 23 & 64 \\
\hline 1 & 8 & 30 & 13 & 36 \\
\hline \multicolumn{5}{|l|}{ Prior adjuvant therapy } \\
\hline Yes & 2 & 7 & 4 & 11 \\
\hline No & 25 & 93 & 32 & 89 \\
\hline \multicolumn{5}{|l|}{ Prior surgery on primary } \\
\hline Yes & 7 & 26 & 9 & 25 \\
\hline No & 20 & 74 & 27 & 75 \\
\hline \multicolumn{5}{|l|}{ Tumor localization } \\
\hline Head & 14 & 52 & 15 & 42 \\
\hline Neck-body & 13 & 48 & 21 & 58 \\
\hline \multicolumn{5}{|l|}{ No. of metastatic sites } \\
\hline 1 & 6 & 22 & 17 & 47 \\
\hline 2 & 12 & 44 & 15 & 42 \\
\hline$\geq 3$ & 9 & 34 & 4 & 11 \\
\hline \multicolumn{5}{|l|}{ Site of metastatic disease } \\
\hline Liver & 20 & 74 & 28 & 78 \\
\hline Lung & 8 & 30 & 9 & 25 \\
\hline Lymph nodes & 18 & 49 & 16 & 45 \\
\hline Peritoneum & 6 & 22 & 8 & 22 \\
\hline Other & 5 & 19 & 0 & 0 \\
\hline \multicolumn{5}{|l|}{ Biliary stenting } \\
\hline Yes & 5 & 19 & 4 & 11 \\
\hline No & 22 & 81 & 32 & 89 \\
\hline \multicolumn{5}{|l|}{ Ca 19.9 baseline } \\
\hline Median & \multirow{2}{*}{\multicolumn{2}{|c|}{$\begin{array}{c}68.3 \\
(0.95-41,251)\end{array}$}} & \multirow{2}{*}{\multicolumn{2}{|c|}{$\begin{array}{c}336.5 \\
(0.80-136,505)\end{array}$}} \\
\hline Range & & & & \\
\hline
\end{tabular}


Table 2. Phase I: dose escalation and DLTs.

\begin{tabular}{|c|c|c|c|c|c|}
\hline Nab-p $\left(\mathrm{mg} / \mathrm{m}^{2}\right)$ & Level & No. Pts & Nab-FOLFIRI (Arm A) & No. pts & Nab-FOLFOX (Arm B) \\
\hline 90 & I & 3 & & 3 & \\
\hline 100 & II & 3 & & 3 & \\
\hline 110 & III & 6 & Liver toxicity G3 (1pt) & 3 & \\
\hline 120 & IV & 6 & MTD & 3 & \\
\hline 130 & $\mathrm{~V}$ & $\begin{array}{c}3+3 \\
\uparrow \downarrow\end{array}$ & $\begin{array}{c}\text { Neutropenia G4, } \\
\text { leucopenia G3 (1 pt) } \\
\text { anemia G3, neutropenia G4, } \\
\text { thrombocytopenia G3, } \\
\text { and mucositis G2 }(1 \mathrm{pt})\end{array}$ & 3 & \\
\hline 140 & VI & 3 & $\begin{array}{c}\text { Neutropenia G4, leucopenia G3, } \\
\text { and thrombocytopenia G3 (1 pt) } \\
\text { Fever G1, asthenia G3, } \\
\text { and hospitalization (1 pt) } \\
\text { DLT }\end{array}$ & 6 & $\begin{array}{c}\text { Mucositis G3, diarrhea G3, } \\
\text { and anorexia G3 (1 pt) }\end{array}$ \\
\hline 150 & VII & NA & & 6 & \\
\hline 160 & VIII & NA & & 6 & $\begin{array}{c}\text { Nausea G3 (1 pt) } \\
\text { MTD }\end{array}$ \\
\hline 170 & IX & $\mathrm{NA}^{*}$ & & 3 & $\begin{array}{c}\text { Sepsis }(1 \mathrm{pt}) \\
\text { Febrile neutropenia G4 and } \\
\text { leucopenia G3 }(1 \mathrm{pt}) \\
\text { DLT }\end{array}$ \\
\hline
\end{tabular}

*NA $=$ not applicable.

\subsection{Phase II}

In phase II (multicenter, randomized 1:1, open label), a total of 84 patients were recruited from November 2015 to January 2017 in 8 centers as reported above. Demographic and baseline disease characteristics of the patients are reported in Table 3 . The median age was 60 years in the Nab-FOLFIRI arm (range 29-75) and 64 years in the Nab-FOLFOX arm (range 47-74). Most of the mPC patients enrolled in the Nab-FOLFIRI and Nab-FOLFOX arms had liver metastases $(79 \%$ and $71 \%)$, a tumor of body/tail of pancreas $(57 \%$ and $55 \%)$, ECOG PS was 0 for 25 patients (60\%) and 29 patients $(69 \%)$, respectively and score 1 for $17(40 \%)$ and $13(31 \%)$ patients. There were fewer patients with previous surgery (10\% vs. $29 \%$ ) and with previous adjuvant therapy ( $2 \%$ vs. $19 \%$ ) in the Nab-FOLFOX arm.

Table 3. Phase II: patient's characteristics.

\begin{tabular}{|c|c|c|c|c|}
\hline \multirow{2}{*}{ Patient's Characteristics } & \multicolumn{2}{|c|}{ Arm A (Nab-FOLFIRI) } & \multicolumn{2}{|c|}{ Arm B (Nab-FOLFOX) } \\
\hline & $N$ & $\%$ & $N$ & $\%$ \\
\hline \multicolumn{5}{|l|}{ Sex } \\
\hline Male & 26 & 62 & 27 & 64 \\
\hline Female & 16 & 38 & 15 & 36 \\
\hline \multicolumn{5}{|l|}{ Age } \\
\hline Median & \multicolumn{2}{|c|}{60 (range 29-75) } & \multicolumn{2}{|c|}{64 (range 47-74) } \\
\hline \multicolumn{5}{|l|}{ ECOG } \\
\hline PS 0 & 25 & 60 & 29 & 69 \\
\hline PS 1 & 17 & 40 & 13 & 31 \\
\hline \multicolumn{5}{|l|}{ Site of primary } \\
\hline Head & 18 & 43 & 19 & 45 \\
\hline Body/tail & 24 & 57 & 23 & 55 \\
\hline
\end{tabular}


Table 3. Cont.

\begin{tabular}{|c|c|c|c|c|}
\hline \multirow{2}{*}{ Patient's Characteristics } & \multicolumn{2}{|c|}{ Arm A (Nab-FOLFIRI) } & \multicolumn{2}{|c|}{ Arm B (Nab-FOLFOX) } \\
\hline & $N$ & $\%$ & $N$ & $\%$ \\
\hline \multicolumn{5}{|l|}{ Previous surgery } \\
\hline Yes & 12 & 29 & 4 & 10 \\
\hline \multicolumn{5}{|l|}{ Previous adjuvant treatment } \\
\hline Yes & 8 & 19 & 1 & 2 \\
\hline Biliary stenting & 7 & 17 & 6 & 14 \\
\hline $\mathrm{M}+$ liver & 33 & 79 & 30 & 71 \\
\hline \multicolumn{5}{|l|}{ Ca 19.9 (U/mL) } \\
\hline median & \multicolumn{2}{|c|}{$\begin{array}{c}566 \\
\text { (range } 3-634,657 \text { ) }\end{array}$} & \multicolumn{2}{|c|}{$\begin{array}{c}1101 \\
\text { (range } 0-167,350)\end{array}$} \\
\hline
\end{tabular}

Among 84 patients, 35 (42\%) completed the planned treatment, 22 in the Nab-FOLFIRI arm and 13 in Nab-FOLFOX. Reasons for study discontinuation were disease progression $(38 \%)$, clinical decision $(6 \%)$, toxicity/adverse events $(19 \%)$, patient's refusal $(2 \%)$, or death $(1 \%)$.

The 1-year survival was $41 \%$ for Nab-FOLFIRI and $50 \%$ for Nab-FOLFOX and the median follow-up was 15.4 and 18.8 months, respectively. PFS in these two regimens were 6.0 months (95\% CI 4.7-8.1) and 5.6 months (95\% CI 4.2-7.2), respectively, (Figure 2), while mOS was 10.2 (95\% CI 8.6-13.3) and 10.4 months (95\% CI 8.4-12.9) (Figure 3). The overall response rate (ORR) was $31 \%$ for both schedules with a clinical benefit rate (CBR) of $69 \%$ and $71 \%$, respectively (Table 4 ).

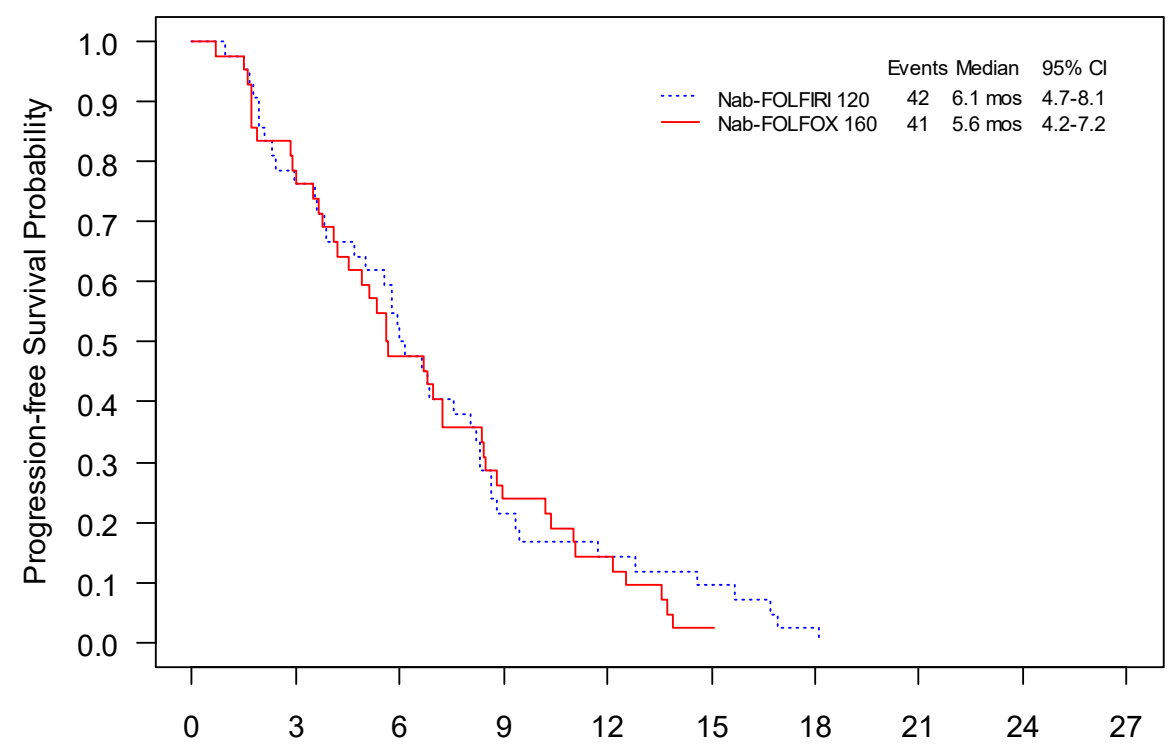

No. at risk

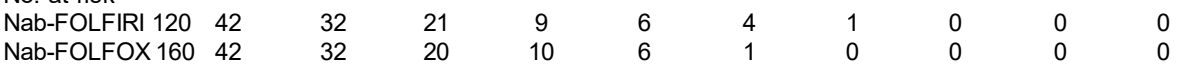

Figure 2. Phase II: progression free survival (PFS) was evaluated using Kaplan-Meier estimates of the median survival times (including $95 \%$ confidence intervals (CI)). 


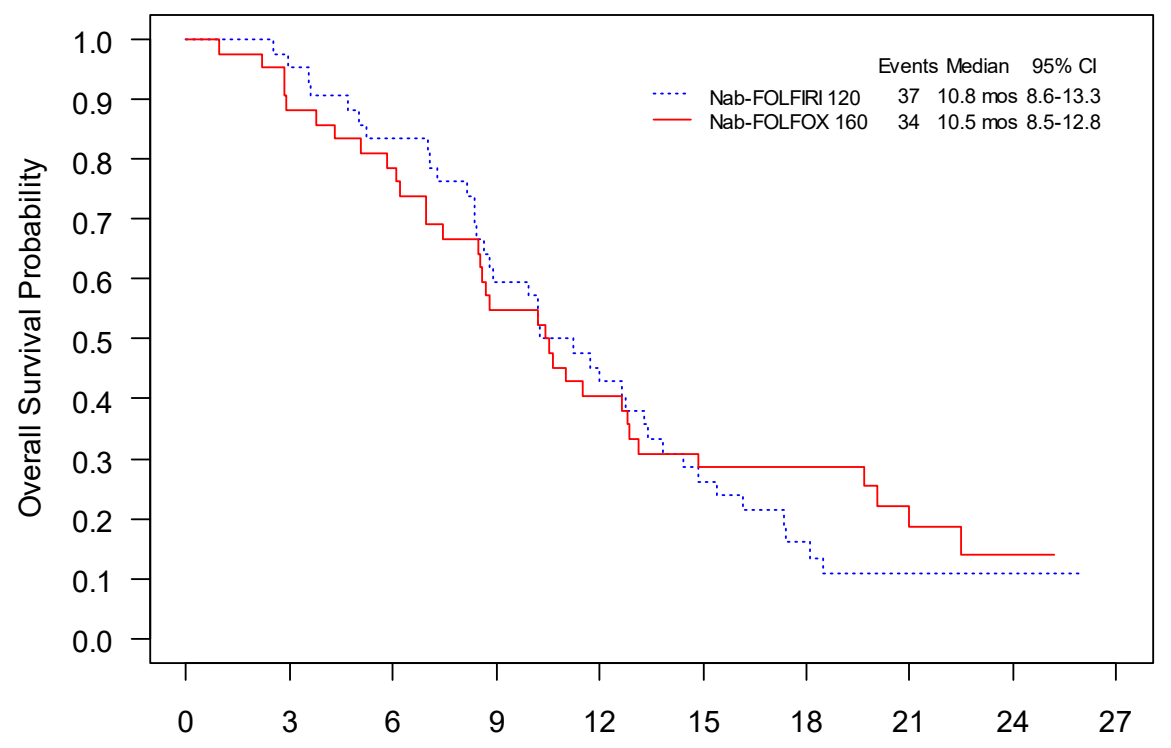

No. at risk

$\begin{array}{lllllllllll}\text { Nab-FOLFIRI 120 } & 42 & 40 & 35 & 25 & 18 & 11 & 6 & 3 & 2 & 0 \\ \text { Nab-FOLFOX 160 } & 42 & 37 & 33 & 23 & 17 & 12 & 11 & 5 & 1 & 0\end{array}$

Figure 3. Phase II: overall survival (OS) was evaluated using Kaplan-Meier estimates of the median survival times (including 95\% confidence intervals (CI)).

Table 4. Phase II: best overall response and clinical benefit rate.

\begin{tabular}{ccccc}
\hline \multirow{2}{*}{ BEST OVERALL RESPONSE } & \multicolumn{2}{c}{ ARM A (Nab-FOLFIRI) } & \multicolumn{2}{c}{ ARM B (Nab-FOLFOX) } \\
\cline { 2 - 5 } & $\boldsymbol{N}$ & $\mathbf{\%}$ & $\boldsymbol{N}$ & $\%$ \\
\hline CR & 0 & 0 & 0 & 0 \\
PR & 13 & 31 & 13 & 31 \\
SD & 16 & 38 & 17 & 40 \\
PD & 11 & 26 & 7 & 17 \\
Not assessed & 2 & 5 & 5 & 71 \\
CLINICAL BENEFIT RATE & & & 30 & 29 \\
CR + PR + SD & 29 & 69 & 12 & \\
Other & 13 & 31 &
\end{tabular}

The most common toxicities of any grade were anemia ( $83 \%$ and 55\%), neutropenia (53\% and $65 \%$ ), nausea (38\%), diarrhea (50\% and $45 \%)$, mucositis (38\% and $31 \%)$, fatigue (55\% and 52\%), and peripheral toxicities (36\% and 71\%). Grade $3-4$ neutropenia occurred in $19 \%$ and $29 \%$, with $12 \%$ and $10 \%$ of febrile neutropenia, respectively (Table 5). Grade $\geq 3$ toxicities in Nab-FOLFIRI were neutropenia (19\%) and febrile neutropenia (12\%). In NabFOLFOX, main grade $\geq 3$ toxicities were neutropenia (29\%), fatigue (14\%), and peripheral neuropathy $(7 \%)$. 
Table 5. Most relevant grade 3 or 4 adverse events and historical controls. NR: not reported.

\begin{tabular}{|c|c|c|c|c|c|c|}
\hline \multicolumn{7}{|c|}{ Phase II: Most Relevant Grade 3 or 4 Adverse Events and Historical Controls. } \\
\hline Event & $\begin{array}{c}\text { Nab-FOLFIRI } \\
n\end{array}$ & $\begin{array}{c}\text { Nab-FOLFIRI } \\
\%\end{array}$ & $\begin{array}{c}\text { Nab-FOLFOX } \\
n\end{array}$ & $\begin{array}{c}\text { Nab-FOLFOX } \\
\%\end{array}$ & $\begin{array}{c}\text { FOLFIRINOX } \\
\text { Conroy, } 2011 \\
n(\%)\end{array}$ & $\begin{array}{c}\text { Nab-FOLFOX } \\
\text { Safran, } 2015 \\
n(\%)\end{array}$ \\
\hline Hematologic & & & & & & \\
\hline Neutropenia & 8 & 19.1 & 12 & 28.6 & 75/164 (45.7) & 6/35 (17.1) \\
\hline Febrile neutropenia & 5 & 11.9 & 4 & 9.5 & $9 / 166(5.4)$ & NR \\
\hline Thrombocytopenia & 1 & 2.4 & 0 & 0 & $15 / 166$ (9.1) & $0 / 35(0.0)$ \\
\hline Anemia & 3 & 7.1 & 4 & 9.5 & $13 / 166(7.8)$ & $2 / 35(5.7)$ \\
\hline \multicolumn{7}{|l|}{ Non hematologic } \\
\hline Fatigue & 2 & 4.8 & 6 & 14.3 & 39/165 (23.6) & $9 / 35(25.7)$ \\
\hline Vomiting & 0 & 0.0 & 0 & 0.0 & 24/166 (14.5) & $0 / 35(0.0)$ \\
\hline Diarrhea & 1 & 2.4 & 0 & 0 & $21 / 165(12.7)$ & $2 / 35(5.7)$ \\
\hline $\begin{array}{c}\text { Sensory } \\
\text { neuropathy }\end{array}$ & 0 & 0 & 3 & 7.1 & $15 / 166(9.0)$ & $2 / 35(5.7)$ \\
\hline Liver toxicity & 2 & 4.8 & 1 & 2.4 & $12 / 165$ (7.3) & $1 / 35(2.9)$ \\
\hline Thromboembolism & 0 & 0.0 & 0 & 0.0 & $11 / 166$ (6.6) & $0 / 35(0.0)$ \\
\hline
\end{tabular}

\subsection{QoL}

The EORTC QLQ-C30 questionnaire was used. Only 35\% and 38\% of patients in Nab-FOLFIRI and Nab-FOLFOX, respectively, completed all of the questionnaires planned at the 6 th administration, and $16 \%$ and $21 \%$ completed all questionnaires during all of the treatments. Therefore, the analyses are only exploratory. Between the two arms, a minor deterioration of global health status was observed in arm A at the 6th cycle (61 baseline vs. 67.8 at 6 th course), similarly to arm B (69.7 at baseline vs. 59.4 at 6 th administration). Improvement in physical and emotional functioning was observed in arm A, along with a decrease in pain, fatigue, nausea, insomnia, anorexia, and constipation. An increase in diarrhea and fatigue was observed in arm B during the first 3 months of CT. The time until $5 \%$ definitive deterioration was not reached in either arms.

\section{Discussion}

PC incidence rates are quickly increasing in developed countries, with half of the patients being metastatic at diagnosis [2]. $\mathrm{MPC}$ is one of the most aggressive and highly lethal cancers, with a median life expectancy of around 1 year with current treatments, and the 5-year OS rate does not exceed 2\% in Europe and the United States [4,5]. Current treatment options for MPC patients include monotherapy or combined therapy. FOLFIRINOX and gem plus Nab-p are both valid first-line options in the metastatic setting of PC [11], and recently, a meta-analysis comparing these two regimens did not provide evidence of a major benefit of one schedule over the other in terms of overall risk of death and progression [12]. A FOLFIRINOX regimen is recommended by ASCO and ESMO guidelines for MPC patients with either 0 or 1 ECOG PS, a favorable comorbidity profile, with good bilirubin values, and a good support system to care for the treatment-related additional toxicity [14]. Nonetheless, it has been primarily tested in relatively young patients and with good performance status. Even in this selected group, high rates of toxicity have been described.

As a consequence, modifications of this regimen have been tested to improve the toxicity profile of FOLFIRINOX. Recently, several studies on a small series of patients have been developed worldwide, testing modified regimens of FOLFIRINOX protocol (so-called modified FOLFIRINOX) in order to reduce toxicity [15] by suppression of the bolus 5-FU and decreasing dose of irinotecan or oxaliplatin [16-20]. Although many retrospective and thorough prospective pilot trials have been reported, no randomized trials evaluating modified FOLFIRINOX have been reported [15]. 
We attempted to develop a regimen triplet that could potentially be better tolerated than FOLFIRINOX in $\mathrm{MPC}$ and hence could be administered as first-line CT for multiple cycles without the development of severe toxicity. In the NabucCO study, FOLFIRINOX was revised by replacing irinotecan or oxaliplatin with Nab-p to obtain two novel schedules (Nab-FOLFIRI and Nab-FOLFOX) that could be as effective and less toxic than the triplet used in the PRODIGE/ACCORD11 study. A rationale to combine platinum, fluoropyrimidine, and a taxane emerges from studies in gastric and head/neck cancer evidencing increased response and survival [21]. In addition, Safran et al. described a phase I NabFOLFOX in advanced PC (both locally advanced and metastatic patients) (MTD Nab-p $150 \mathrm{mg} / \mathrm{m}^{2}$ ), and a phase II trial is still ongoing [22]. Nab-p is a target CT with a more selective action that uses nanotechnology and shows a good efficacy and safety profile in mPC $[23,24]$. In phase I, we determined MTD and DLTs of Nab-p to demonstrate that NabFOLFIRI and Nab-FOLFOX are feasible and reasonably tolerated. In phase II, we achieved an ORR of $31 \%$ for both regimens with a CBR of $69 \%$ and $71 \%$ for Nab-FOLFIRI and Nab-FOLFOX, respectively. Despite the limitations of a comparison between different trials, we can state that the results in terms of RR are similar to data reported by Conroy and colleagues [8] and better than the meta-analysis of retrospective and phase II studies by Thibodeau et al. [25] that reported an overall pooled RR of $24.5 \%$ (95\% CI: $16.92-32.15 \%)$. The median PFS (6 and 5.6 months) and OS (10.2 and 10.4 months) in our trial are similar to those reported in the PRODIGE4/ACCORD11 trial $[8,24]$.

The NabucCO trial was designed with eligibility criteria quite similar to the Conroy study [8] in order to enroll comparable populations. The median age of patients in the NabucCO study was 60 in the Nab-FOLFIRI arm, 64 in the Nab-FOLFOX arm, and 61 in the FOLFIRINOX trial. The site of the primary tumor was mainly the head of the pancreas in NabucCO phase II with 43\% (18/42) and 45\% (19/42), 17\% (7/42) and 14\% (6/42) of biliary stenting, and 79\% (33/42) and 71\% (30/42) of patients with hepatic metastasis for Nab-FOLFIRI and Nab-FOLFOX, respectively. In the PRODIGE4/ ACCORD11 trial, $39.2 \%$ of patients had a primary tumor in the head of the pancreas, $14 \%$ of them had a biliary stenting at the time of enrollment, and $87 \%$ had hepatic metastasis. Overall, these data suggest that the NabucCO population had an important percentage of unfavorable factors that could have worsened the safety of treatment in line with the PRODIGE4/ACCORD11 population.

In the NabucCO phase II trial, the median cycles of treatment were 9 (range 1 to 12) and 8 (range 1 to 12) for Nab-FOLFIRI and Nab-FOLFOX, respectively, suggesting a good compliance to therapy. In the PRODIGE4/ACCORD11 trial, the median of cycles was 10, while Abrams et al. reported a median duration of chemotherapy with FOLFIRINOX of 100 days (7 cycles) in a U.S.-based cohort of 609 patients with mPC [25].

With regard to hematologic G3/4 toxicity, neutropenia is the most common AE in our regimens, with 19.1 and 28.6 in Nab-FOLFIRI and Nab-FOLFOX, respectively; this rate is significantly lower than that reported in the FOLFIRINOX trial (45.7\% G3/4 neutropenia) [8].

In the Conroy trial, the occurrence of severe diarrhea (12.7\%) and vomiting (14.5\%) during the first cycles of treatment had a negative impact on the overall quality of life of $\mathrm{mPC}$ patients [8]. Notably, among non-hematologic toxicity in both of our studies, diarrhea was strongly reduced $(2.4 \%$ and $0.0 \%)$ and vomiting was not registered; again, our schedules compare favorably with FOLFIRINOX in terms of gastrointestinal AE.

Nab-p nowadays is not registered in Europe for use over first-line of treatment, and triplet is used only in a subgroup of patients with good PS and rarely in successive settings due to the rapid deterioration of clinical conditions of patients after first-line treatment. Therefore, NabucCO regimens could represent potential treatments in order to give a possibility to use either triplets and Nab-p as first-line CT. On the whole, both the activity and tolerability of both proposed triplet regimens is promising, with a low rate and severity of AEs. Therefore, Nab-FOLFIRI and Nab-FOLFOX might be promising first-line 
$\mathrm{CT}$ options for $\mathrm{mPC}$ patients, though further studies are required to explore the potential of these novel triplets in phase III settings.

Author Contributions: E.G. and F.D.C. designed the study; E.G., E.M., V.V., E.R., C.V., G.T., L.T., G.G., C.L. and T.P.L. enrolled patients and collected data; L.B. analyzed data; S.P. and L.A. revised the manuscript; E.G., S.P., and L.A. wrote the paper. All authors have read and agreed to the published version of the manuscript.

Funding: Support by GOIRC and Celgene International Sarl, contract IIT33321_AX0103_NABUCCO Ver05-29 oct13.

Institutional Review Board Statement: The study was conducted according to the guidelines of the Declaration of Helsinki, and approved by Comitato Etico Area Vasta Centro (protocol code 42176/2013, 16 December 2013).

Informed Consent Statement: Informed consent was obtained from all subjects involved in the study.

Data Availability Statement: Data available on request to the corresponding author.

Conflicts of Interest: The authors declare no conflict of interest, and the funders had no role in the design of the study; in the collection, analyses, or interpretation of data; in the writing of the manuscript, or in the decision to publish the results.

\section{References}

1. Ilic, M.; Ilic, I. Epidemiology of pancreatic cancer. World J. Gastroenterol. 2016, 22, 9694-9705. [CrossRef] [PubMed]

2. Siegel, R.L.; Miller, K.D.; Jemal, A. Cancer Statistics, 2017. CA Cancer J. Clin. 2017, 67, 7-30. [CrossRef] [PubMed]

3. Malvezzi, M.; Carioli, G.; Bertuccio, P.; Rosso, T.; Boffetta, P.; Levi, F.; La Vecchia, C.; Negri, E. European cancer mortality predictions for the year 2016 with focus on leukaemias. Ann. Oncol. 2016, 27, 725-731. [CrossRef]

4. Bosetti, C.; Bertuccio, P.; Malvezzi, M.; Levi, F.; Chatenoud, L.; Negri, E.; La Vecchia, C. Cancer mortality in Europe, 2005-2009, and an overview of trends since 1980. Ann. Oncol. 2013, 24, 2657-2671. [CrossRef]

5. Sohal, D.P.; Mangu, P.B.; Khorana, A.A.; Shah, M.A.; Philip, P.A.; O’Reilly, E.M.; Uronis, H.E.; Ramanathan, R.K.; Crane, C.H.; Engebretson, A.; et al. Metastatic Pancreatic Cancer: American Society of Clinical Oncology Clinical Practice Guideline. J. Clin. Oncol. 2016, 34, 2784-2796. [CrossRef] [PubMed]

6. Sultana, A.; Smith, C.T.; Cunningham, D.; Starling, N.; Neoptolemos, J.P.; Ghaneh, P. Meta-Analyses of Chemotherapy for Locally Advanced and Metastatic Pancreatic Cancer. J. Clin. Oncol. 2007, 25, 2607-2615. [CrossRef]

7. Heinemann, V.; Haas, M.; Boeck, S. Systematic treatment of advanced pancreatic cancer. Cancer Treat Rev. 2013, 38, 843-853. [CrossRef] [PubMed]

8. Conroy, T.; Desseigne, F.; Ychou, M.; Bouché, O.; Guimbaud, R.; Bécouarn, Y.; Adenis, A.; Raoul, J.-L.; Gourgou-Bourgade, S.; De La Fouchardière, C.; et al. FOLFIRINOX versus Gemcitabine for Metastatic Pancreatic Cancer. N. Engl. J. Med. 2011, 364, 1817-1825. [CrossRef] [PubMed]

9. Ibrahim, N.K.; Desai, N.; Legha, S.; Soon-Shiong, P.; Theriault, R.L.; Rivera, E.; Esmaeli, B.; Ring, S.E.; Bedikian, A.; Hortobagyi, G.N.; et al. Phase I and pharmacokinetic study of ABI-007, a Cremophor-free, protein-stabilized, nanoparticle formulation of paclitaxel. Clin. Cancer Res. 2002, 8, 1038-1044. [PubMed]

10. Von Hoff, D.D.; Ervin, T.; Arena, F.P.; Chiorean, E.G.; Infante, J.; Moore, M.; Seay, T.; Tjulandin, S.A.; Ma, W.W.; Saleh, M.N.; et al. Increased survival in pancreatic cancer with nab-paclitaxel plus gemcitabine. N. Engl. J. Med. 2013, 369, 1691-1703. [CrossRef] [PubMed]

11. Kim, S.; Signorovitch, J.E.; Yang, H.; Patterson-Lomba, O.; Xiang, C.Q.; Ung, B.; Parisi, M.; Marshall, J.L. Comparative Effectiveness of nab-Paclitaxel Plus Gemcitabine vs FOLFIRINOX in Metastatic Pancreatic Cancer: A Retrospective Nationwide Chart Review in the United States. Adv. Ther. 2018, 35, 1564-1577. [CrossRef]

12. Pusceddu, S.; Ghidini, M.; Torchio, M.; Corti, F.; Tomasello, G.; Niger, M.; Prinzi, N.; Nichetti, F.; Coinu, A.; Di Bartolomeo, M.; et al. Comparative Effectiveness of Gemcitabine plus Nab-Paclitaxel and FOLFIRINOX in the First-Line Setting of Metastatic Pancreatic Cancer: A Systematic Review and Meta-Analysis. Cancers 2019, 11, 484. [CrossRef]

13. Eisenhauer, E.A.; Therasse, P.; Bogaerts, J.; Schwartz, L.H.; Sargent, D.; Ford, R.; Dancey, J.; Arbuck, S.; Gwyther, S.; Mooney, M.; et al. New response evaluation criteria in solid tumours: Revised RECIST guideline (version 1.1). Eur. J. Cancer 2009, 45, 228-247. [CrossRef]

14. Ducreux, M.; Cuhna, A.S.; Caramella, C.; Hollebecque, A.; Burtin, P.; Goéré, D.; Seufferlein, T.; Haustermans, K.; Van Laethem, J.L.; Conroy, T.; et al. Cancer of the pancreas: ESMO Clinical Practice Guidelines for diagnosis, treatment and follow-up. Ann. Oncol. 2015, 26 (Suppl. 5), v56-v68. [CrossRef]

15. Lambert, A.; Gavoille, C.; Conroy, T. Current status on the place of FOLFIRINOX in metastatic pancreatic cancer and future directions. Ther. Adv. Gastroenterol. 2017, 10, 631-645. [CrossRef] [PubMed] 
16. Mahaseth, H.; Brutcher, E.; Kauh, J.; Hawk, N.; Kim, S.; Chen, Z.; Kooby, D.A.; Maithel, S.K.; Landry, J.; El-Rayes, B.F. Modified FOLFIRINOX Regimen with Improved Safety and Maintained Efficacy in Pancreatic Adenocarcinoma. Pancreas 2013, 42, 1311-1315. [CrossRef] [PubMed]

17. Blazer, M.; Wu, C.; Goldberg, R.M.; Phillips, G.; Schmidt, C.; Muscarella, P.; Wuthrick, E.; Williams, T.M.; Reardon, J.; Ellison, E.C.; et al. Neoadjuvant modified (m) FOLFIRINOX for locally advanced unresectable (LAPC) and borderline resectable (BRPC) adenocarcinoma of the pancreas. Ann. Surg. Oncol. 2014, 22, 1153-1159. [CrossRef]

18. Ghorani, E.; Wong, H.H.; Hewitt, C.; Calder, J.; Corrie, P.; Basu, B. Safety and Efficacy of Modified FOLFIRINOX for Advanced Pancreatic Adenocarcinoma: A UK Single-Centre Experience. Oncology 2015, 89, 281-287. [CrossRef]

19. Li, X.; Guo, C.; Li, Q.; Wei, S.; Zhang, Q.; Chen, Y.; Shen, Y.; Ma, T.; Li, G.; Gao, S.; et al. Association of Modified-FOLFIRINOXRegimen-Based Neoadjuvant Therapy with Outcomes of Locally Advanced Pancreatic Cancer in Chinese Population. Oncologist 2018, 24, 301-e93. [CrossRef]

20. Wang, Z.-Q.; Zhang, F.; Deng, T.; Zhang, L.; Feng, F.; Wang, F.-H.; Wang, W.; Wang, D.-S.; Luo, H.-Y.; Xu, R.-H.; et al. The efficacy and safety of modified FOLFIRINOX as first-line chemotherapy for Chinese patients with metastatic pancreatic cancer. Cancer Commun. 2019, 39, 26-28. [CrossRef] [PubMed]

21. Vermorken, J.B.; Remenar, E.; Van Herpen, C.; Gorlia, T.; Mesia, R.; Degardin, M.; Stewart, J.S.; Jelic, S.; Betka, J.; EORTC 24971/TAX 323 Study Group; et al. Cisplatin, Fluorouracil, and Docetaxel in Unresectable Head and Neck Cancer. N. Engl. J. Med. 2007, 357, 1695-1704. [CrossRef] [PubMed]

22. Safran, H.; Charpentier, K.P.; Perez, K.; Mantripragada, K.; Miner, T.; DiPetrillo, T.; Kuritzky, B.; Apor, E.; Bishop, K.; Luppe, D.; et al. FOLFOX+Nab-Paclitaxel (FOLFOX-A) for Advanced Pancreatic Cancer: A Brown University Oncology Research Group Phase I Study. Ann. J. Clin. Oncol. 2016, 39, 619-622.

23. Braiteh, F.S.; Patel, M.B.; Parisi, M.; Ni, Q.; Park, S.; Faria, C. Comparative effectiveness and resource utilization of nab-paclitaxel plus gemcitabine vs FOLFIRINOX or gemcitabine for the first-line treatment of metastatic pancreatic adenocarcinoma in a US community setting. Cancer Manag. Res. 2017, 9, 141-148. [CrossRef] [PubMed]

24. Thibodeau, S.; Voutsadakis, I.A. FOLFIRINOX Chemotherapy in Metastatic Pancreatic Cancer: A Systematic Review and Meta-Analysis of Retrospective and Phase II Studies. J. Clin. Med. 2018, 7, 7. [CrossRef] [PubMed]

25. Abrams, T.A.; Meyer, G.; Meyerhardt, J.A.; Wolpin, B.M.; Schrag, D.; Fuchs, C.S. Patterns of Chemotherapy Use in a U.S.-Based Cohort of Patients with Metastatic Pancreatic Cancer. Oncologist 2017, 22, 925-933. [CrossRef] 